\title{
Editorials
}

\section{How accurate are GPs at integrating evidence into prescribing decisions?}

\section{EVIDENCE IN THE CONSULTATION}

A 56-year-old attends his GP to discuss his newly diagnosed hypertension and asks the GP about the benefits and harms of taking long-term hypotensive medication ... How accurate are we (GPs, healthcare professionals and other specialists) in providing this information?

GPs are unlikely to have fingertip evidence knowledge for management decisions, but if we don't, should we? Treadwell et al' highlight GP inaccuracy in numerical assessment of benefits and harms of longterm medications used in several long-term conditions. The General Medical Council's Good Medical Practice ${ }^{2}$ states that doctors should:

keep up to date... [and] prescribe drugs or treatments, including repeat prescriptions, only when you have adequate knowledge of the patient's health and are satisfied that the drugs or treatment serve the patient's needs.

Also, to:

provide effective treatments based on the best available evidence.

Within consultations we commonly find two opportunities for evidence consideration. One is at diagnosis; for instance, calculating the probability of deep vein thrombosis using Wells score, risk assessment for primary prevention of coronary heart disease and stroke (for example, QRisk33), or arranging investigations. The second is in the discussion of management decisions including prescribing, for example, benefits and harms of anticoagulation in atrial fibrillation and heart attack, and ischaemic stroke prevention using $\mathrm{CHA}_{2} \mathrm{DS}_{2}$-VASc and HAS-BLED. 4,5 This second opportunity is the most explicit in providing figures of condition risk in UK primary care; other long-term conditions may not have supportive risk scores.

Therefore, decision making requires clinicians to identify the evidence required;

\section{GPs [need to] change knowledge bases in pace with evidence, especially in the modern era of novel medications and rapid information transfer.}

what clinical question are we asking on behalf of our particular patient at that time? This may be diagnosis, prognosis, investigations, or therapeutics. Shared decision-making with patients requires reliable medical evidence, and the need to improve provision is increasingly recognised. The authors of this editorial are Chairs of the Royal College of General Practitioners (RCGP) overdiagnosis group, ${ }^{6}$ the members of which are keen to reduce unnecessary treatments and harm reduction for patients. Choose Wisely UK, ${ }^{7}$ hosted by the Academy of Medical Royal Colleges, suggests doctors use the acronym BRAN - What are the Benefits? What are the Risks? What are the Alternatives? What if I do Nothing? in management discussions. Rethinking Medicine $^{8}$ accepts that resources, rather than all-knowing, is the way forward. This group explores the evolution of the biomedical model into psychosocial and personalised contexts of individual patient care. They state:

tomorrow's professionals will also need to consider the evolving context in which medicine operates and need to keep less information in their heads but know how to access, navigate, synthesise and utilise information in order to provide the best care for individual patients.

\section{ACCESS TO EVIDENCE}

Many GPs admit to low confidence in current knowledge assessment, as highlighted in Treadwell et al.' Once the MRCGP is under our belt we lack the luxury of study budgets (though educational events are tax-deductible) and are cast into the 'do-it-yourself' continual professional development (CPD) for appraisal decades lexcept for some mandatory training of dubious priorityl. This may be totally appropriate, but does notensure generations of GPs change knowledge bases in pace with evidence, especially in the modern era of novel medications and rapid information transfer. We don't think it feasible for most primary care clinicians, including GPs, to be expert number crunchers and interpreters of evidence. How can we cope?

Well, most of us rely on guideline creators to do the maths for us. Having all the evidence, including that not published, is important for guideline creators, and we support AllTrials (https://www.alltrials. net) in trying to achieve this to create best evidence bases. Reported evidence is fortunately undergoing a sea change away from relative risk, which inflates benefits, to absolute risk and using numbers needed to treat (NNT) or harm (NNH). In many trials, all-cause mortality is reported, as well as disease-related outcomes to evaluate long-term efficacy. The guideline developer the National Institute for Health and Care Excellence (NICE), produces patient decision aids (commonly used as GP decision aids in our experiencel for NNT and NNH. NICE guidance nowadays frequently incorporates tables of advantages and disadvantages of investigations and management options, for an example see guidelines for diagnosis and management of prostate cancer (NG131). There are other sites that provide guidelines and websites of evidence bases, which GPs can place on clinic desktops. We like TheNNT (https://www.thennt.com) for its brevity and speed and My Studies (https:// www.mystudies.org) for more in-depth study investigation. The Cochrane library (https:// www.cochrane.orgl provides plain language summaries of its systematic reviews.

\section{THE FUTURE OF KEEPING EVIDENCE UP} TO DATE

There is a need for trustworthy, robust guideline creators to continue to manage and interpret evidence at understandable levels to clinical non-statistical specialists: 


\section{ADDRESS FOR CORRESPONDENCE}

\section{Jane Wilcock}

Silverdale Medical Practice, Swinton, Salford M27 8HP, UK.

Email: Jane.wilcockanhs.net

Dajanewilcock we recommend ballpark figures of benefit and harms rounded to nearest whole numbers. Computer-based decision support to integrate specific patient measures with wider evidence bases to help in decision making is a developing area. ${ }^{10}$ Digital NHS could have a role working with primary care to link potential benefits and harms more easily onto clinic screens, providing one GP resource at the point of prescribing. Using data repositories smartly to give relevant evidence at the right moment is key. Perhaps the British National Formulary (https://bnf.nice.org.uk) could include numerical evidence and link to GP prescribing seamlessly. In addition, ongoing efficacy for an individual patient, for example, trends and set interval reporting of blood pressure, haemoglobin A1c, weight reduction, or adverse effects like renal deterioration, could be highlighted to encourage deprescribing where benefit is lacking or adverse effects occur.

Longer-term and out-of-clinics educators should provide numerical evidence as standard so that assimilation occurs through familiarity. The RCGP should take a lead as they have numerous online learning opportunities, with Essential Knowledge Updates ${ }^{11}$ covering much of our curriculum. Ensuring evidence is embedded onto courses at a national, faculty, and online level, and guiding GPs and their allied clinicians to manageable, prioritised updates, while keeping the touch light to allow for personal interests, may be a way forward.
We are in an era of time contraction, increasing patient complexity, polypharmacy, and multimorbidity, with an expansion of new medicines and increasing primary care research. Sharing relevant data with all of us, including busy GPs head down and patient-facing all week, is paramount. Patients trust us and expect good advice. The study by Treadwell et al ${ }^{1}$ demonstrates a need to improve on current evidence resources available to primary care prescribers at a national level.

\section{Jane Wilcock,}

GP, Silverdale Medical Practice, Salford; Chair, Overdiagnosis Interest Group, Royal College of General Practitioners, London.

\section{Katharine Alsop,}

GP, Nightingale Valley Practice, Bristol; Vice-chair, Overdiagnosis Interest Group, Royal College of General Practitioners, London.

\section{David Spitzer,}

GP, Cranwich Road Surgery; Vice-chair, Overdiagnosis Interest Group, Royal College of General Practitioners, London.-

\section{Provenance}

Freely submitted; externally peer reviewed.

\section{Competing interests}

The authors have declared no competing interests.

DOI: https://doi.org/10.3399/bjgp20X708857

\section{REFERENCES}

1. Treadwell JS, Wong G, Milburn-Curtis C, et al. GPs' understanding of the benefits and harms of treatments for long-term conditions: an online survey. BJGP Open 2020; DOI: https:// doi.org/10.3399/bjgpopen20X101016.

2. General Medical Council. Domain 1: knowledge skills and performance. In: Good medical practice. 2019. https://www.gmc-uk.org/ethicalguidance/ethical-guidance-for-doctors/goodmedical-practice laccessed 24 Feb 2020).

3. ClinRisk. Welcome to the $\mathrm{QRISK}^{\circledR} 3-2018$ risk calculator. 2018. https://qrisk.org/three/index. php laccessed 24 Feb 2020).

4. Lip G. CHA2DS-VASc score for atrial fibrillation stroke risk. MD\&CALC. https://www.mdcalc com/cha2ds2-vasc-score-atrial-fibrillationstroke-risk (accessed 25 Feb 2020).

5. Pisters R. HAS-BLED score for major bleeding risk. MD\&CALC. https://www.mdcalc.com/hasbled-score-major-bleeding-risk laccessed 25 Feb 2020).

6. Royal College of General Practitioners. Overdiagnosis group. RCGP, 2020. https:// www.rcgp.org.uk/clinical-and-research/about/ special-interest-groups/overdiagnosis-group. aspx (accessed 24 Feb 2020).

7. Choosing Wisely UK. About Choosing Wisely UK. https://mww.choosingwisely.co.uk/aboutchoosing-wisely-uk (accessed 24 Feb 2020).

8. Rethinking Medicine. Why we are rethinking medicine. NHS England and RCGP. Supported by Kaleidoscope Health \& Care, 2018. https://www.rethinkingmedicine. co.uk/uploads/8/0/2/1/80213224/ rethinkingmedicineposition.pdf laccessed 24 Feb 2020).

9. National Institute for Health and Care Excellence. Prostate cancer: diagnosis and management. NG131. 2019. https://www.nice. org.uk/guidance/ng131 (accessed 24 Feb 2020).

10. Souza NM, Sebaldt RJ, Mackay JA, et al. CCDSS Systematic Review Team. Computerized clinical decision support systems for primary preventive care: a decision-maker-researcher partnership systematic review of effects on process of care and patient outcomes. Implement Sci2011; 6 : 87.

11. Royal College of General Practitioners Learning. Essential Knowledge Updates \& Challenges. RCGP. https://elearning.rcgp.org. uk/course/index. php? categoryid=2 laccessed 24 Feb 2020). 